\title{
Prenatal Stress and Psychiatric Symptoms During Early Phases of the COVID-I 9 Pandemic in Italy
}

\author{
Chiara Colli (iD) \\ Chiara Penengo $\mathbb{D}$ \\ Marco Garzitto ${ }^{1}$ \\ Lorenza Driul $^{2}$ \\ Alessia Sala ${ }^{2}$ \\ Matilde Degano ${ }^{2}$ \\ Heidi Preis ${ }^{3}$ \\ Marci Lobel ${ }^{3}$ \\ Matteo Balestrieri \\ 'Unit of Psychiatry, Department of \\ Medicine (DAME), University of Udine, \\ Udine, Italy; ${ }^{2}$ Obstetric-Gynaecologic \\ Clinic, Department of Medicine (DAME), \\ University of Udine, Udine, Italy; \\ ${ }^{3}$ Department of Psychology, Stony Brook \\ University, Stony Brook, New York, NY, \\ I I794, USA
}

Correspondence: Chiara Colli

Clinica Psichiatrica, Ospedale S.M.

Misericordia, ASUFC, Piazzale Santa Maria

Della Misericordia, 15, Udine, 33100, Italy

Tel +390432559284

Email colli.chiara9!@gmail.com
Purpose: In February 2020, Italy became the first European country to face the coronavirus disease 2019 (COVID-19) outbreak. The concerns of infection, financial worries, loss of freedom, and isolation during the ongoing pandemic can lead to negative psychological effects, including anxiety, depression, and obsessive-compulsive disorder. The main aim of our study was to evaluate the relationship between pandemic-related stress and pregnancyspecific stress and assess their role in the development of psychiatric symptoms. We predicted that pregnancy-specific stress would mediate an association of pandemic-related stress with psychiatric symptoms.

Patients and Methods: A total of 258 pregnant women were assessed for general emotional symptoms with the General Anxiety Disorder-7 (GAD-7), Patient Health Questionnaire-2 (PHQ2), and an Obsessive-Compulsive Disorder screening (OCD). The Revised Prenatal Distress Questionnaire (NuPDQ) and the Pandemic-Related Pregnancy Stress Scale (PREPS) were administered as measures of pregnancy-specific stress (PSS and pandemic-related, respectively). Mediation effects by NuPDQ for PREPS stress scales on psychiatric outcomes were calculated, using regression series and correcting for general covariates.

Results: Almost a third of the sample reported clinically relevant anxiety levels $(32.6 \%)$, $11.2 \%$ were positive for OCD screening and less than $5 \%$ were positive for depression screening. The stress related to feeling unprepared for delivery and postpartum (PREPS-PS) predicted PHQ-2 score, both directly and indirectly via PSS, and it predicted GAD-7 score only indirectly. The stress related to fear of infection (PREPS-PIS) was directly associated to GAD-7 score and - through PSS - to PHQ-2 score and OCD.

Conclusion: The pandemic onset contributed to poor mental health, especially anxiety, in a substantial portion of Italian pregnant women. Our results emphasize the importance of strategies to reduce pregnancy-specific stress, as well as to diminish stress due to the pandemic. Identifying risk factors for psychological suffering is important to prevent potential long-term consequences for mothers and their offspring.

Keywords: COVID-19 pandemic, pregnancy-specific stress, anxiety, depression, obsessivecompulsive disorder

\section{Introduction}

In February 2020, Italy became the first European country to face the Coronavirus Disease 2019 (COVID-19) outbreak. The contagion rapidly spread and, by April 28th 2020, 199,470 people were known to have been infected in Italy. ${ }^{1}$ Several measures were adopted to limit the spread of the virus and a national lockdown was imposed on March 11th. Then, following a substantial reduction of new cases, restrictions were removed on July $3 \mathrm{rd}$, although personal protective equipment and social distancing were still mandatory. 
Alongside the COVID-19 pandemic, a psychiatric epidemic was reported. The possible repercussion of this unusual situation on mental health was identified as a crucial problem since the pandemic onset; in fact, in March 2020, the World Health Organization (WHO) developed recommendations to support psychological well-being during the pandemic. ${ }^{2}$ The psychological consequences of the COVID-19 outbreak have become a global challenge, particularly for highly vulnerable individuals. Hence, there is a need to identify these population groups and guarantee them appropriate health-care provision. $^{3}$

Pregnant women are considered at high-risk for severe impact of the pandemic because of their increased susceptibility to distress. ${ }^{4}$ Knowledge about SARS-COV2 infection and its complications during pregnancy was initially limited and much of it was derived from studies regarding two other coronaviruses, MERS and SARS. There had been no cases of intrauterine maternal-foetal transmission with SARS and MERS, so the likelihood of vertical transmission for SARS-COV2 was considered low. ${ }^{5}$ At the time of our study, there was a lack of definitive evidence about whether the SARS-COV2 virus may create life-threatening clinical conditions for pregnant women and increase risk of preterm delivery, other adverse birth outcomes, or neonatal morbidity and mortality. ${ }^{6,7}$ Uncertainty about the potential impacts of the virus and the lack of reliable information are sources of stress that may adversely affect the well-being of mothers and the health and development of their children. $^{8}$

Furthermore, pregnant women seem to be more vulnerable to anxiety and depression compared to the general population $^{9,10}$ and they are also at risk to develop obsessive-compulsive disorder (OCD). ${ }^{11}$ Indeed, several studies have detected an uptick in levels of anxiety and depression in expectant women during the COVID-19 outbreak; ${ }^{12-14}$ these results are in line with previous studies of mothers-to -be during natural disasters. ${ }^{15}$ Also, obsessive-compulsive symptoms, which are more common during pregnancy as oppose to other time periods ${ }^{11}$ are likely to be more prevalent during the COVID-19 pandemic because of infection fears and public health measures emphasizing protective behaviours such as handwashing. ${ }^{16}$

Stress-related to the pandemic co-occurs with pregnancy-specific stress (PSS), which derives from worries about physical symptoms, bodily changes, and concerns about the baby. ${ }^{17,18}$ It is well known that PSS can negatively affect birth outcome and development of the child and some evidence demonstrating an association of PSS with clinically relevant anxiety and depressive symptoms. ${ }^{19,20}$

\section{Problem Statement}

Our aim was to assess the role of PSS role together with pandemic-related stress in determining the presence of a selected psychiatric symptomatology. We predicted that pandemic-related stress would be associated with elevated PSS, which in turn would predict higher risk of depression, anxiety, and OCD. In other words, we predicted that PSS would mediate an association of pandemic-related stress with psychiatric symptomatology.

\section{Patients and Methods}

A cross- sectional study was carried out to evaluate the pregnancy specific stress, pandemic related stress and clinical outcome in a sample of pregnant women.

\section{Participants}

Data were collected between April 22nd and August 31st 2020. This time period encompasses the first (AprilMay) and second phases (July-August) of the pandemic onset in Italy. Pregnant women were recruited among those receiving outpatient prenatal care at the Gynaecology Clinic of the University Hospital of Udine. The participation in the research was proposed directly by the participant's physician. Inclusion criteria were current pregnancy, age over 18 years, and Italian fluency. Two hundred fifty-eight women agreed to participate and completed the study questionnaires. All recruited women were volunteers and received no incentives for their participation. The participants filled out the questionnaire in a paper-and-pencil format at the hospital during routine clinical check-ups. All participants provided informed consent. All procedures performed in this study were in accordance with the ethical standards of the 1964 Helsinki Declaration and its subsequent amendments. Approval was granted by the Medical Ethics Committee of Friuli Venezia Giulia region (CEUR-2018-Sper-027-ASUIUD). Written informed consent was obtained from all participants included in the study. The privacy and dignity of the respondents were assured by making all the information anonymous and confidential. 


\section{Measures}

Data were collected with a questionnaire that gathered background information, COVID-19 exposure, and pregnancy and prenatal care aspects. We also investigated knowledge of the COVID-19 pandemic with a scale consisting of two questions ('I feel knowledgeable about COVID-19' and 'I believe I can control not getting COVID-19'). Data concerning pregnancy risk-status were checked by gynaecologists who followed the women during pregnancy (S.A. and D.M.). Additionally, we administered the following self-report instruments to measure stress and psychiatric symptoms.

To evaluate stress among pregnant women related to the COVID-19 pandemic, we used the Pandemic-Related Pregnancy Stress Scale (PREPS). ${ }^{21}$ The instrument includes 15 items that are scored on a scale from 1 ("Very Little") to 5 ("Very Much"). It comprises three internally consistent scales: Preparedness Stress (PS, 7 items), Prenatal Infection Stress (PIS, 5 items), and Positive Appraisal (PA, 3 items). The PREPS-PS is related to feeling unprepared for delivery and postpartum, PREPS-PIS evaluates the stress related to fear of perinatal infection, and PREPS-PA assesses strategies for coping with pandemic-related stress. ${ }^{21,22}$ In this study we focused on PREPS stress scales and we only included PREPS-PA as possible covariate. We used the Italian version of the PREPS adapted by our research group (Penengo et al, 2021 accepted), that showed acceptable-to-good internal consistency for PS $(\alpha=0.760)$, PIS $(\alpha=0.857)$, and PA $(\alpha=0.747)$ scales.

To assess PSS, we used a revised version of the Prenatal Distress Questionnaire (NuPDQ), developed by Lobel and colleagues. ${ }^{23}$ The instrument comprises 17 items ranging from 0 ("Never") to 2 ("Very Often"); the total score is the sum of each item and range from 0 to 34 . This instrument showed good reliability $(\alpha=0.55-0.79)$ in various studies. ${ }^{18}$ We used the Italian version validated by our group. ${ }^{24}$

Anxiety symptoms were evaluated through the General Anxiety Disorder-7 (GAD-7). ${ }^{25}$ A total score of seven or above is considered a clinically meaningful level of anxiety symptoms during pregnancy. ${ }^{26}$ The instrument showed good internal consistency $(\alpha=0.89),{ }^{26}$ and was well validated in pregnant women. ${ }^{27}$

To screen symptoms of major depressive disorder, we used the Patient Health Questionnaire-2 (PHQ-2), a short form of the PHQ- 9 questionnaire. ${ }^{28,29}$ A score of 3 or above is considered the cut-off for depression. The internal consistency of PHQ-2 resulted good $(\alpha=0.83),{ }^{30}$ and it resulted to be an efficient screening tool for depression in pregnant women. ${ }^{31}$

Lastly, to assess the presence or absence of obsessivecompulsive symptoms, we used the OCD Screening, derived from the Structured Clinical Interview for DSM$5,{ }^{32}$ which consists of two questions. The screening assesses the presence of obsessive-compulsive symptoms when women responded "Yes" to both of the questions.

\section{Data Analysis}

We considered potential covariates of the psychiatric symptomatology outcome variables in various domains: positive coping, as measured with PREPS (PA scale, 15); pregnancy status (i.e., pregnancy has been defined as at-risk during clinical check-ups, no/yes; in the 3rd pregnancy trimester, no/yes; first child, no/yes); COVID-19 pandemic (three measures; i.e., assessment conducted in the 2nd Italian pandemic phase, no/yes; level of perceived knowledge about pandemic, 1-5; having had rescheduled appointments in prenatal care, no/yes); general personal information (two measures; i.e., age in years; education in schooling years); financial status (three measures; i.e., not being currently employed, no/yes; financial status, low/ medium/high; having suffered from recent loss of income, no/yes); general well-being (seven measures; i.e., history of emotional/psychiatric problems, no/yes; having experienced emotional/physical abuse, no/yes; having a chronic medical condition, no/yes; level of perceived support from family/friend, 1-5; level of perceived support from partner, $1-5$; level of healthy activities carried out, $1-5$; living alone, no/yes).

All the covariates were used to fit a complete regression model for each outcome (i.e., GAD-7 score, 0-21; PHQ-2 score, 0-6; OCD positive screening, no/yes). Then, backward selections based on Akaike's Information Criterion were adopted to identify a set of significant covariates for each outcome. Further analyses were replicated using both complete and selected covariates and without covariates. For regression analyses, model statistical significance, parameter estimates, and the coefficient of determination $\left(\mathrm{R}^{2}\right.$ or McFadden's pseudo- $\mathrm{R}^{2}$ ) were calculated. Variance inflation factors below the square root of 2 were accepted.

A series of mediation analyses were conducted to estimate the mediation of NuPDQ in the regressions of psychiatric symptoms on the two PREPS stress scales. 
Estimates were made using a non-parametric bootstrapping method (with 10,000 replication samples). ${ }^{33}$ Average Causal Mediation Effect (ACME) was calculated as a measure of indirect effect, together with percent mediated. Average Direct Effect (ADE) and total effect were also calculated. Only models with a statistically significant association between the independent variable (PREPS) and both the dependent variables (GAD-7; PHQ-2; OCD) and mediator (NuPDQ) were considered evidence of mediation.

Mean-substitution was preferred to manage missing data in multivariate analyses. All analyses were conducted using R-4.0.3. ${ }^{34}$

\section{Results}

\section{Sample Characteristics}

There was a similar percentage of women in different pandemic phases (1st: April-May 2020/2nd: July-August 2020), pregnancy periods (Early: first and second trimesters/Late: third trimester), and pregnancy risk-levels (Low-risk/Highrisk; Fisher's exact test for count data, all with: OR $\leq 1.519$, $\mathrm{p} \geq 0.140$ ). Frequencies are reported in Table 1 .

The mean age of participants was $32.5 \pm 5.12$ years, the mean years of education were $15.1 \pm 3.60$; most women were married or in a stable cohabiting relationship (251 women, $99.2 \%$ ). $37.9 \%$ of the participants lived in centres of more than 50,000 inhabitants, and $88.0 \%$ of them had access to the outdoors during the period of pandemicrelated movement restrictions. The majority of the sample were Caucasian women (95.7\%). Only two women were

Table I Total Sample (N=258). Distributions by 2020 Pandemic Phase in Italy, Pregnancy Period and Pregnancy Risk Status

\begin{tabular}{|l|l|c|c|c|}
\hline & & \multicolumn{3}{|c|}{ Pandemic } \\
\hline Trimester & Risk & Ist Phase & 2nd Phase & Total \\
\hline \multirow{2}{*}{ Early (i/ii) } & Low-risk & $5 \mathrm{I}$ & 54 & 105 \\
& High-risk & 22 & 20 & 42 \\
& All & 73 & 74 & 147 \\
\hline \multirow{2}{*}{ Late (iii) } & Low-risk & 32 & 37 & 69 \\
& High-risk & 15 & 27 & 42 \\
& All & 47 & 64 & 111 \\
\hline \multirow{2}{*}{ All } & Low-risk & 83 & 91 & 174 \\
& High-risk & 37 & 47 & 84 \\
& All & 120 & 138 & 258 \\
\hline
\end{tabular}

Notes: Ist phase, Pandemic, first phase (April-May 2020); 2nd phase, Pandemic, second pandemic phase (July-August 2020); Early (i/ii), First and second trimester of pregnancy; Late (iii), Third trimester of pregnancy; High-risk, high-risk pregnancy; Low-risk, low-risk pregnancy. diagnosed with COVID-19 and eight others had any indirect contact with the disease. Sociodemographic and general characteristics of the sample are reported in Table 2, together with participants' scores on study instruments.

$32.6 \%$ of participants scored above the cut-off for anxiety on the GAD-7 (i.e., score $\geq 7$, without differences between pandemic phases $(\mathrm{p}=0.780)$, period of pregnancy $(\mathrm{p}=0.394)$, or level of pregnancy risk $(\mathrm{p}=0.456)$. In comparison, only $4.8 \%$ of the sample had high scores for depression (PHQ-2 score $\geq 3$ ), also without any differences between pandemic phases $(\mathrm{p}=0.555)$, period of pregnancy $(p=0.766)$, or pregnancy risk $(p=1.000)$. Finally, $11.2 \%$ of the sample had a positive score on the obsessivecompulsive screening, also without any differences between the three variable groups (pandemic phases $\mathrm{p}=0.548$; period of pregnancy $\mathrm{p}=0.544$; pregnancy risk $\mathrm{p}=0.056)$.

There were few differences in other participant characteristics between those in the first or second phases of the pandemic. Compared to women who participated in the first phase, a greater portion of participants in the second phase reported below-average financial status (18.7\% vs 8.1\%; $\mathrm{p}=0.025 ; \mathrm{OR}=2.590)$ and fewer reported average financial status $(77.6 \%$ vs $88.3 \%, p=0.042$; $\mathrm{OR}=0.461$. Moreover, there were few differences between women with high-risk and low-risk pregnancies: the mean age was higher for women with high-risk pregnancies $(p=0.014)$ and they also reported more frequent chronic medical conditions $(p<0.001)$. Lastly, we found that a greater portion of women in the 3rd trimester of pregnancy reported high financial status $(\mathrm{p}=0.046)$, and a higher level of pregnancy-specific stress $(p=0.008)$ than women earlier in pregnancy. Women having their first child, were more often younger $(p=0.004)$, more frequently employed $(\mathrm{p}=0.022)$, and showed a greater level of healthy activity $(\mathrm{p}=0.014)$.

\section{Multiple Linear/Logistic Regressions}

Table 3 reports the list of selected covariates for each outcome (detailed results are reported in supplementary materials; Tables S1 and S2). The GAD-7 model was statistically significant $\left(\mathrm{R}^{2}=0.203\right.$, adjusted to 0.135 ; $\left.\mathrm{F}_{19,222}=2.982, \mathrm{p}<0.001\right)$ and four predictors were backward-selected $\left(\mathrm{R}^{2}=0.178\right.$, adjusted to $0.164 ; \mathrm{F}_{4237}=12.84$, $\mathrm{p}<0.001)$. Similarly, the PHQ- 2 model $\left(\mathrm{R}^{2}=0.174\right.$, adjusted to $\left.0.105 ; \mathrm{F}_{19,228}=2.53, \mathrm{p}=0.001\right)$ resulted in selection of five predictors $\left(\mathrm{R}^{2}=0.150\right.$, adjusted to $0.133 ; \mathrm{F}_{5242}=8.57$, $\mathrm{p}<0.001)$. Therefore, mediation analyses were conducted 
Table 2 Total Sample $(\mathrm{N}=258)$. Sociodemographic and Clinical Description. Continuous Measures are Reported in Part A. Binary Measures are Reported in Part B

\begin{tabular}{|c|c|c|}
\hline Part A. Continuous Measures [Range] & N. Patients (\%) & Mean \pm SD \\
\hline Age in years $[18-46]$ & $255(98.8 \%)$ & $32.5 \pm 5.12$ \\
\hline Schooling in years[3-24] & $226(87.6 \%)$ & $15.1 \pm 3.60$ \\
\hline Level of stress $[1-5]$ & $250(96.9 \%)$ & $2.3 \pm 0.98$ \\
\hline Level of support from family and friends [1-5] & $246(95.3 \%)$ & $4.1 \pm 1.16$ \\
\hline Level of support from partner $[1-5]$ & $252(97.7 \%)$ & $4.5 \pm 0.88$ \\
\hline Level of healthy activities[ $[-5]$ & $252(97.7 \%)$ & $3.7 \pm 0.93$ \\
\hline Knowledge about Covid-19 [I-5] & $249(96.5 \%)$ & $3.3 \pm 1.01$ \\
\hline Number of children $[0-5]$ & $249(96.5 \%)$ & $0.6 \pm 0.79$ \\
\hline GAD-7 [0-2I] & $242(93.8 \%)$ & $5.9 \pm 4.20$ \\
\hline PHQ-2 [0-6] & $248(96.1 \%)$ & $1.0 \pm 1.20$ \\
\hline NuPDQ [0-34] & $244(94.6 \%)$ & $1 \mathrm{I} .4 \pm 5.00$ \\
\hline PREPS-PS [I-5] & $243(94.2 \%)$ & $2.9 \pm 0.86$ \\
\hline PREPS-PIS [I-5] & $245(95.0 \%)$ & $2.6 \pm 1.02$ \\
\hline PREPS-PA [1-5] & $245(95.0 \%)$ & $2.2 \pm 1.06$ \\
\hline Part B. Binary measures & & Count (\%) \\
\hline Currently working & $254(98.4 \%)$ & $187(73.6 \%)$ \\
\hline Low financial status & $245(95.0 \%)$ & $34(13.9 \%)$ \\
\hline Medium financial status & 245 (95.0\%) & $202(82.4 \%)$ \\
\hline High financial status & 245 (95.0\%) & $9(3.7 \%)$ \\
\hline Loss of income due to pandemic & $246(95.3 \%)$ & 77 (3I.3\%) \\
\hline Chronic medical condition & $254(98.4 \%)$ & $44(17.3 \%)$ \\
\hline Emotional/Physical abuse & $252(97.7 \%)$ & $4(1.6 \%)$ \\
\hline History of Emotional/Psychiatric problems & $25 \mathrm{I}(97.3 \%)$ & $9(3.6 \%)$ \\
\hline Lives alone & $25 I(97.3 \%)$ & II (4.4\%) \\
\hline High anxiety (GAD-7>6) & $242(93.8 \%)$ & 79 (32.6\%) \\
\hline High depression (PHQ-2>2) & $248(96.1 \%)$ & $12(4.8 \%)$ \\
\hline Positive screening for OCD & $249(96.5 \%)$ & $28(11.2 \%)$ \\
\hline
\end{tabular}

Abbreviations: GAD-7, General Anxiety Disorder - 7; NuPDQ, Revised Prenatal Distress Questionnaire; OCD, Obsessive-Compulsive problems; PA, PREPS, Positive Appraisal scale; PHQ-2, Patient Health Questionnaire - 2; PIS, PREPS, Perinatal Infection Stress scale; PREPS, Pandemic-Related Pregnancy Stress questionnaire; PS, PREPS, Preparedness Stress scale; SD, standard deviation.

using selected covariates for GAD-7 and PHQ-2 scores. The OCD model was not statistically significant (pseudo$\mathrm{R}^{2}=0.150 ; \chi_{19}^{2}=26.25, \mathrm{p}=0.123$ ), hence the mediation analysis for positive OCD screening was conducted without covariates.

\section{Mediation Analysis}

Figures 1-3 show the analyses of the effects (ADE and ACME) of the pandemic-related stress scales (PREPS-PS and PREPS-PIS), with the mediation of NuPDQ, on the psychometric scales, as described in the methods section (detailed results are reported in supplementary materials; $\underline{\text { Tables S3 }}$ and S4).

The GAD-7 score was significantly predicted $(\mathrm{p} \leq 0.001)$ by all the stress measures after correcting for selected covariates (NuPDQ: $\quad \beta=+0.294, R^{2}=0.259 ; \quad$ PREPS-PS: $\beta=+0.197$,
$\mathrm{R}^{2}=0.216$; PREPS-PIS: $\left.\beta=+0.216, \mathrm{R}^{2}=0.224\right)$. When included as a mediator (Figure 1), NuPDQ completely mediated the effect of PREPS-PS (50.2\% [20.05\%, 135.37\%]; ADE: $\mathrm{p}=0.133$ ) and partially mediated the effect of PREPS-PIS (33.6\% [14.30\%, 74.26\%]).

Similarly, with selected covariates, all the stress measures statistically significantly predicted the PHQ-2 score (NuPDQ: $\beta=+0.273, R^{2}=0.220$; PREPS-PS: $\beta=+0.273$, $\mathrm{R}^{2}=0.196$; PREPS-PIS: $\beta=+0.159, \mathrm{R}^{2}=0.175$ ). For PHQ-2 (Figure 2), the NuPDQ score partially mediated the effect of PREPS-PS $(39.8 \%[15.19 \%, 94.01 \%])$ and totally that of PREPS-PIS $(45.2 \%, \quad[16.86 \%, 140.67 \%], \quad$ ADE: $\mathrm{p}=0.134$ ).

Finally, a positive screening for OCD was predicted by NuPDQ $\left(\mathrm{OR}=1.80, \mathrm{p}=0.003\right.$, pseudo- $\mathrm{R}^{2}=0.051$, $\left.\chi_{1}^{2}=8.87, \quad \mathrm{p}=0.003\right) \quad$ and PREPS-PIS $\quad(\mathrm{OR}=1.80$, 
Table 3 Multiple Linear Regressions with GAD-7 Scores and PHQ-2 Scores as Dependent Variables. Standardized Data and Coefficients are Reported with Their 95\% Confidence Interval. See Table SI and S2 for Details

\begin{tabular}{|c|c|c|}
\hline Measure & GAD-7 & PHQ-2 \\
\hline & $\beta \pm 95 \% \mathrm{Cl}$ & $\beta \pm 95 \% \mathrm{Cl}$ \\
\hline Intercept & $+4.324[-1.186,+9.834]$ & $+1.966 *[+0.406,+3.526]$ \\
\hline PREPS-PA & $-0.032[-0.565,+0.502]$ & $+0.052[-0.101,+0.204]$ \\
\hline At-risk pregnancy & $+0.865[-0.281,+2.0111]$ & $+0.002[-0.326,+0.330]$ \\
\hline Late pregnancy ( $3 r d$ trimester) & $-0.098[-1.161,+0.964]$ & $+0.158[-0.146,+0.461]$ \\
\hline Ist child & $+0.019[-1.063,+1.101]$ & $-0.101[-0.413,+0.210]$ \\
\hline 2nd pandemic phase & $+0.292[-0.788,+1.372]$ & $-0.095[-0.403,+0.213]$ \\
\hline Knowledge about COVID-19 & $+0.698^{*}[+0.154,+1.242]^{\S}$ & $+0.167 *[+0.011,+0.324]^{\S}$ \\
\hline Rescheduled prenatal care appointment & $-0.219[-1.600,+1.161]$ & $-0.185[-0.582,+0.212]$ \\
\hline Age (years) & $+0.038[-0.076,+0.152]$ & $-0.008[-0.040,+0.025]$ \\
\hline Schooling (years) & $+0.052[-0.113,+0.218]$ & $+0.001[-0.046,+0.049]$ \\
\hline Not employed & $+0.469[-0.857,+1.796]$ & $+0.417^{*}[+0.039,+0.794]^{\S}$ \\
\hline Low financial status & $-0.309[-1.869,+1.252]$ & $-0.197[-0.647,+0.253]$ \\
\hline Loss of income due to pandemic & $+0.059[-1.077,+1.196]$ & $+0.162[-0.163,+0.487]$ \\
\hline History of emotional/psychiatric problem & $+6.923 *[+4.075,+9.771]^{\S}$ & $+1.479 *[+0.652,+2.306]^{\S}$ \\
\hline Emotional/Physical abuse & $-0.130[-4.360,+4.100]$ & $-0.182[-1.411,+1.046]$ \\
\hline Chronic medical condition & $-0.871[-2.303,+0.560]$ & $-0.294[-0.708,+0.121]^{\S}$ \\
\hline Level support from family/friends & $-0.309[-0.788,+0.171]$ & $-0.044[-0.180,+0.093]$ \\
\hline Level support from partner & $+0.330[-0.314,+0.974]$ & $-0.067[-0.246,+0.112]$ \\
\hline Level healthy activities & $-0.958^{*}[-1.529,-0.386]^{\S}$ & $-0.268^{*}[-0.434,-0.103]^{\S}$ \\
\hline Lives alone & $+3.015^{*}[+0.404,+5.626]^{\S}$ & $+0.392[-0.366,+1.150]$ \\
\hline
\end{tabular}

Notes: *Statistically significant with $\mathrm{p}<0.050$. ${ }^{\S}$ Predictor maintained in the corresponding backward-selected model.

Abbreviations: Cl, confidence interval; GAD-7, General Anxiety Disorder, 7-items, questionnaire; PHQ-2, Patient Health Questionnaire, 2-items, questionnaire; PREPSPA, Pandemic-Related Pregnancy Stress questionnaire, Positive Appraisal scale.

$\mathrm{p}=0.006$, pseudo- $\left.\mathrm{R}^{2}=0.046, \chi^{2}=8.09, \mathrm{p}=0.004\right)$ scores, but not by PREPS-PS. NuPDQ (Figure 3) completely mediated the effect of PREPS-PIS on OCD screening $(25.2 \%,[1.89 \%, 94.51 \%]$, ADE: $\mathrm{p}=0.053)$.

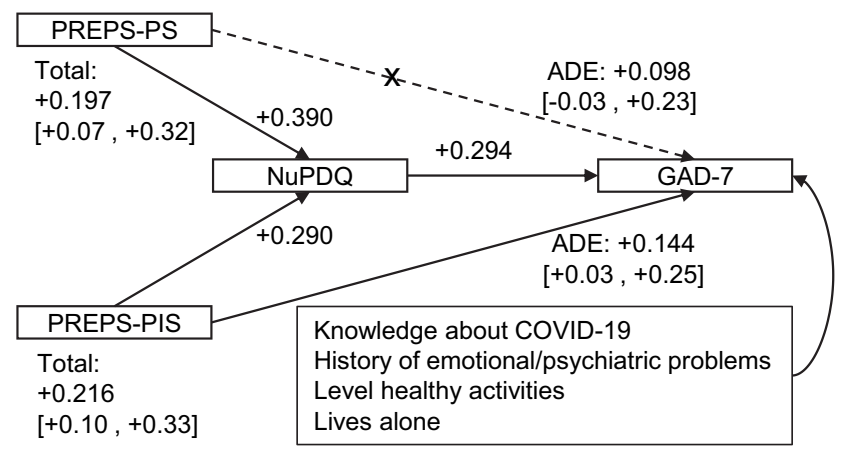

Figure I Mediations of pregnancy-specific stress (NuPDQ) in regressions of anxiety score (GAD-7) on pandemic-related stress measures (PREPS-PS and PREPSPIS). Mediation coefficients are reported with $95 \%$ confidence interval between square brackets. Selected covariates are listed. See Tables SI and S4 for details. Abbreviations: ADE, Average Direct Effect; GAD-7, General Anxiety Disorder, 7-items, questionnaire; NuPDQ, Revised Prenatal Distress Questionnaire; PIS, Prenatal Infection Stress scale; PREPS, Pandemic-Related Pregnancy Stress Scale; PS, Preparedness Stress scale; $X$, Arrow not statistically significant after mediation.

\section{Discussion}

This study illustrates the psychological experience of pregnant women at the onset of the COVID-19 pandemic in Italy. Almost a third of the sample reported anxiety levels compatible with a clinical disorder (32.6\% of the sample), and a relevant percentage was positive for screening obsessive-compulsive problems (11.2\%). In contrast, less than $5 \%$ of the sample had clinically relevant depression scores. The observed frequencies suggest that the sample is mainly characterized by anxiety problems. However, depression and anxiety are typically comorbid during pregnancy, and high anxiety is a risk factor for antenatal depression. ${ }^{35}$ Study findings suggest that a global pandemic may create unique circumstances that result in different patterns and prevalence of psychopathology among pregnant women, with particular impact manifested in their anxiety. ${ }^{36-38}$ About half of the participants that were positive for obsessive-compulsive symptoms also indicated anxiety problems. The frequency of high anxiety 


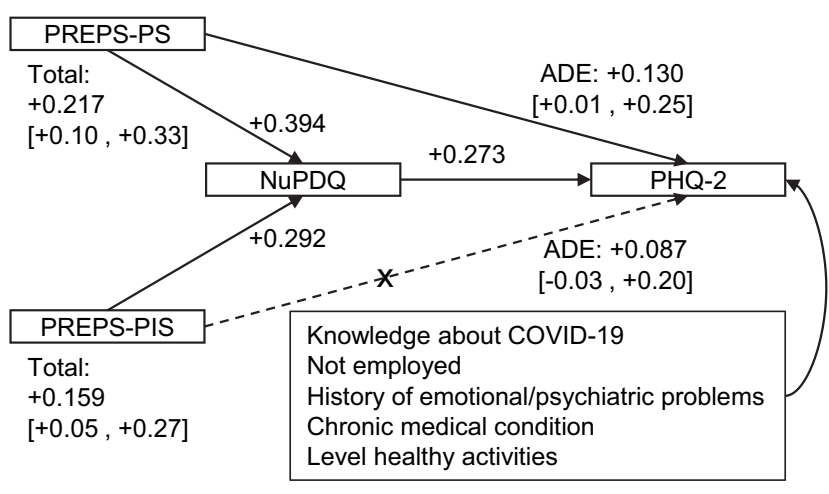

Figure 2 Mediations of pregnancy-specific stress (NuPDQ) in regressions of depression score (PHQ-2) on pandemic-related stress measures (PREPS-PS and PREPS-PIS). Mediation coefficients are reported with $95 \%$ confidence interval between square brackets. Selected covariates are listed. See Tables S2 and S4 for details.

Abbreviations: ADE, Average Direct Effect; NuPDQ, Revised Prenatal Distress Questionnaire; PHQ-2, Patient Health Questionnaire, 2-items; PIS, Prenatal Infection Stress scale; PREPS, Pandemic-Related Pregnancy Stress Scale; PS, Preparedness Stress scale; $X$, arrow not statistically significant after mediation.

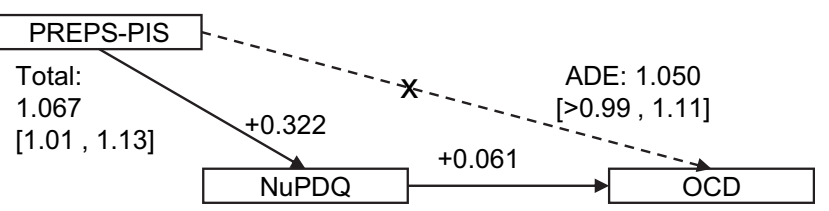

Figure 3 Mediations of pregnancy-specific stress (NuPDQ) in regressions of screening for obsessive-compulsive problems on stress for prenatal infection (PREPS-PIS). Mediation coefficients are reported with $95 \%$ confidence interval between square brackets.

Abbreviations: ADE, Average Direct Effect; NuPDQ, Revised Prenatal Distress Questionnaire; OCD, positive screening for Obsessive-Compulsive Disorder; PIS, Prenatal Infection Stress scale; PREPS, Pandemic-Related Pregnancy Stress Scale; $X$, Arrow not statistically significant after mediation.

was also higher than that reported for the Italian general population during the pandemic: Mazza and colleagues found high anxiety levels in $7.2 \%$ of their sample, and very high anxiety levels in $11.5 \%{ }^{39}$

A recent meta-analysis assessed the psychological impact of COVID 19 pandemic in pregnant women. It included 19 papers from ten different countries and showed an increased prevalence of anxiety and depression among expectant mothers, with an overall reported rate of $42 \%$ and $25 \%$ respectively. ${ }^{40}$

Moreover, a longitudinal evaluation of pregnant women during quarantine showed a more pronounced increase in anxiety, depression and negative affect compared to non-pregnant women, suggesting that being pregnant may be consider a risk factor for psychopathological consequences of the pandemic. ${ }^{41}$

Samples recruited in the first and second pandemic onset phases were quite homogeneous. Nevertheless, a few differences emerged between April-May and JulyAugust. For example, we found lower financial status among participants in the second phase. Low socioeconomic status is a well-known risk factor for psychological disorders, in particular, depression. ${ }^{42,43}$ Studies of the SARS outbreak in $2003^{44}$ demonstrated that income loss resulting from quarantines and lockdowns, when people are unable to work, can be a risk factor for poor mental health, ${ }^{45}$ especially depressive symptoms. ${ }^{46}$ Consistent with these findings, in our sample, current unemployment was related to clinical levels of depression. We also detected increased levels of anxiety in unpartnered women. Living alone is a significant determinant of loneliness, a condition that can increase the risk of mental impairment leading to anxiety, depression, and even suicidal ideation. ${ }^{47}$

Higher levels of anxiety and depression were also associated with previous emotional or psychiatric problems. A recent Italian study detected a greater level of COVID19 concerns and anxiety among expectant mothers with a history of psychiatric disturbances. ${ }^{48}$ Similarly, many studies have found that a history of psychiatric illness increases the risk of antenatal onset of depression. ${ }^{49}$

Lastly, knowledge about COVID-19 was positively related to a higher level of anxiety and depression, a result in contrast with several studies that associated the absence of information about COVID-19 infection and pregnancy with a greater level of anxiety and distress among childbearing women. ${ }^{36,50,51}$ Nevertheless, our data need to be understood in the context of the uncertainty and constantly evolving knowledge about the mechanism of transmission of the virus and its potential consequences that were typical during the onset of the pandemic when this study was conducted. ${ }^{52}$

Apart from the factors that were associated with poorer mental health, we found that healthy activities seem to have a protective role against anxiety and depression symptoms, also as shown in previous research. ${ }^{53,54}$ In particular, many studies in the general population and in pregnant women report a positive impact of healthy behavior on depressive symptoms, corroborating the beneficial relationship between healthy lifestyles and lower levels of depression that we found. ${ }^{53,55,56}$

As we predicted, stress related to the pandemic cooccurred with stress that is unrelated to the pandemic but focused on pregnancy itself. Both types of stress were potent predictors of poorer mental health, with distinct 
patterns of association for each of the three outcomes. For example, women experiencing greater stress involving fears about perinatal COVID-19 infection had higher anxiety, and this association occurred in part because these women also experienced greater distress unassociated with the pandemic, specific to being pregnant. Previous studies concerning the SARS outbreak in $2003^{57,58}$ similarly showed that fear of becoming infected was related to greater anxiety in pregnant women. Additionally, in the present study, women who reported higher pandemicrelated stress involving concerns that they would be unprepared for childbirth or the postpartum also experienced greater anxiety; this association was entirely explained by the association of this type of pandemic-related stress with pandemic unrelated, pregnancy-specific stress (PSS). Similarly, PSS entirely explained the association of perinatal infection stress with depressive symptoms and partially explained the association of preparedness stress with this mental health outcome. Feeling unprepared for birth or postpartum due to the pandemic can lead to demoralization and hopelessness and hence to depressive symptoms. $^{59}$

Lastly, obsessive-compulsive symptoms were also predicted by higher stress involving fears of perinatal infection, and this association was explained by higher PSS among women who reported this type of pandemicrelated stress. This result parallels a previous study ${ }^{16}$ that found in a sample of university students that the fear of COVID-19 infection was strongly correlated with scores on a measure of OCD, suggesting that such fear, together with anxiety and pandemic-induced quarantine, is a risk factor for obsessive-compulsive symptoms.

Given the pivotal role of PSS in mediating many of the associations of pandemic-related stress with anxiety, depressive, and obsessive-compulsive symptoms, study results suggest that it is essential not only to reduce stressful conditions related to the pandemic, but also to alleviate the pregnancy-specific conditions that create distress for women whether in times of a pandemic or not.

\section{Limitations and Strengths}

A major limitation of this study is the cross-sectional design, thus we cannot confirm causal relationships between measures. However, we did find scientifically plausible associations using well-validated instruments, in many cases corroborating findings of comparable research. Another strength is the evaluation of multiple dimensions of mental health and numerous possible risk factors. The current pandemic has created numerous stressors for pregnant women, whose physical and psychological vulnerability underscores the need to examine a variety of potential mental health consequences and the range of risk factors that may affect them.

\section{Conclusion}

The present study highlights how the COVID-19 pandemic is influencing the mental health of Italian expectant mothers and indicates that a substantial portion of them are experiencing high anxiety. In our analysis, pandemic-related stress predicts the development of anxiety, depressive, and obsessive-compulsive symptoms. These associations are partially mediated by experiencing more of the type of stress that pregnant women commonly experience, which includes stress related to their concerns about their health and changes to their body, about impending delivery, and about the challenges they are about to face as the parent of a new-born. Hence, our results emphasize the importance of strategies to reduce such pregnancy-specific stress, as well as to diminish the stress that has arisen for pregnant women due to the COVID-19 pandemic. Anxiety, depression, and OCD symptoms in pregnant women have been shown to elevate risk for a variety of adverse maternal, foetal, and infant outcomes. ${ }^{60-65}$ Identifying risk factors for poor mental health and promptly intervening is fundamental to stem detrimental consequences in the short term, as well as to prevent their longer term harms to women and children.

\section{Disclosure}

The authors report no conflicts of interest in this work.

\section{References}

1. Riccardo F, Andrianou X, Bella A, et al. Epidemia COVID-19 Aggiornamento Nazionale 28 Aprile 2020. 2020.

2. World Health Organization. Mental Health and Psychosocial Considerations During COVID-19 Outbreak. World Health Organization. 1-6. 2020.

3. Hossain MM, Tasnim S, Sultana A, et al. Epidemiology of mental health problems in COVID-19: a review. F1000Research. 2020;9:1-16. doi:10.12688/f1000research.24457.1

4. Saccone G, Florio A, Venturella R, et al. Psychological Impact of Coronavirus Disease 2019 in Pregnant Women. Am J Obstetrics Gynecol. 2020. doi:10.1016/j.ajog

5. Schwartz DA, Graham AL. Potential maternal and infant outcomes from coronavirus 2019-NCOV (SARS-CoV-2) infecting pregnant women: lessons from SARS, MERS, and other human coronavirus infections. Viruses. 2020;12(2):1-16. doi:10.3390/v12020194 
6. Hantoushzadeh S, Shamshirsaz AA, Aleyasin A, et al. Maternal death due to COVID-19. Am J Obstet Gynaecol. 2020;223(1):109.e1-109. e16. doi:10.1016/j.ajog.2020.04.030

7. Zaigham M, Andersson O. Maternal and perinatal outcomes with COVID-19: a systematic review of 108 pregnancies. Acta Obstet Gynaecol Scand. 2020;99(7):823-829. doi:10.1111/aogs.13867

8. Thapa SB, Mainali A, Schwank SE, Acharya G. Maternal mental health in the time of the COVID-19 pandemic. Acta Obstet Gynaecol Scand. 2020;99(7):817-818. doi:10.1111/aogs.13894

9. Sinesi A, Maxwell M, O’Carroll R, Cheyne H. Anxiety scales used in pregnancy: systematic review. BJPsych Open. 2019;5(1):1-13. doi:10.1192/bjo.2018.75

10. Howard LM, Molyneaux E, Dennis CL, Rochat T, Stein A, Milgrom J. Non-psychotic mental disorders in the perinatal period. Lancet. 2014;384(9956):1775-1788. doi:10.1016/S0140-6736(14) 61276-9

11. Russell EJ, Fawcett JM, Mazmanian D. Risk of obsessive-compulsive disorder in pregnant and postpartum women: a meta-analysis. J Clin Psychiatry. 2013;74(4):377-385. doi:10.4088/ JCP.12r07917

12. Berthelot N, Lemieux R, Garon-Bissonnette J, Drouin-Maziade C, Martel E, Maziade M. Uptrend in distress and psychiatric symptomatology in pregnant women during the coronavirus disease 2019 pandemic. Acta Obstet Gynaecol Scand. 2020;99(7):848-855. doi:10.1111/aogs.13925

13. Moyer CA, Compton SD, Kaselitz E, Muzik M. Pregnancy-related anxiety during COVID-19: a nationwide survey of 2740 pregnant women. Arch Womens Ment Health. 2020;23(6):757-765. doi:10.1007/s00737-020-01073-5

14. Mappa I, Distefano FA, Rizzo G. Effects of coronavirus 19 pandemic on maternal anxiety during pregnancy: a prospectic observational study. J Perinat Med. 2020;48(6):545-550. doi:10.1515/jpm-20200182

15. Fatema SR, Islam MS, East L, Usher K. Women's health-related vulnerabilities in natural disasters: a systematic review protocol. BMJ Open. 2019;9(12):1-5. doi:10.1136/bmjopen-2019-032079

16. Ji G, Wei W, Yue KC, et al. Effects of the COVID-19 pandemic on obsessive-compulsive symptoms among university students: prospective cohort survey study. J Med Internet Res. 2020;22(9):1-13. doi: $10.2196 / 21915$

17. Hamilton JG, Lobel M. Types, patterns, and predictors of coping with stress during pregnancy: examination of the Revised Prenatal Coping Inventory in a diverse sample. J Psychosom Obstet Gynaecol. 2008;29(2):97-104. doi:10.1080/01674820701690624

18. Alderdice F, Lynn F, Lobel M. A review and psychometric evaluation of pregnancy-specific stress measures. J Psychosom Obstet Gynaecol. 2012;33(2):62-77. doi:10.3109/0167482X.2012.673040

19. Lobel M, Dunkel Schetter C. Pregnancy and Prenatal Stress. Encycl Ment Heal. 2016;3:318-329. doi:10.1016/B978-0-12-3970459.00164-6

20. Ibrahim SM, Lobel M. Conceptualization, measurement, and effects of pregnancy-specific stress: review of research using the original and revised Prenatal Distress Questionnaire. J Behav Med. 2020;43 (1):16-33. doi:10.1007/s10865-019-00068-7

21. Preis H, Mahaffey B, Lobel M. Psychometric properties of the Pandemic-Related Pregnancy Stress Scale (PREPS). J Psychosom Obstet Gynaecol. 2020;41(3):191-197. doi:10.1080/ 0167482X.2020.1801625

22. Preis H, Mahaffey B, Heiselman C, Lobel M. Vulnerability and resilience to pandemic-related stress among U.S. women pregnant at the start of the COVID-19 pandemic. Soc Sci Med. 2020;266:1-4 doi:10.1016/j.socscimed.2020.113348

23. Lobel M, Cannella DL, Graham JE, DeVincent C, Schneider J, Meyer BA. Pregnancy-Specific Stress, Prenatal Health Behaviors, and Birth Outcomes. Heal Psychol. 2008;27(5):604-615. doi: $10.1037 / \mathrm{a} 0013242$
24. Penengo C, Colli C, Garzitto M, Driul L, Cesco M, Balestrieri M. Validation of the Italian version of the Revised Prenatal Coping Inventory (NuPCI) and its correlations with pregnancy-specific stress. BMC Pregnancy Childbirth. 2020;20:1-14. doi:10.1186/ s12884-020-03159-5

25. Spitzer RL, Kroenke K, Williams JBW, Löwe B, Brief A. Measure for Assessing Generalized Anxiety Disorder The GAD-7. Arch Intern Med. 2006;166:1092-1097. doi:10.1001/archinte.166.10.1092

26. Zhong QY, Gelaye B, Zaslavsky AM, et al. Diagnostic validity of the generalized anxiety disorder - 7 (GAD-7) among pregnant women. PLoS One. 2015;10(4):1-17. doi:10.1371/journal.pone.0125096

27. Gong Y, Zhou H, Zhang Y, et al. Validation of the 7-item Generalized Anxiety Disorder scale (GAD-7) as a screening tool for anxiety among pregnant Chinese women. J Affect Disord. 2021;1:282.

28. Spitzer RL, Kroenke K, Williams JBW. Validation and Utility of a Self-report Version of PRIME-MD. The PHQ Primary Care Study. JAMA. 1999;282:1737-1744. doi:10.1001/jama.282.18.1737

29. Kroenke K, Spitzer RL, Williams JBW. The Patient Health Questionnaire-2 Validity of a Two-Item Depression Screener. Med Care. 2003;41:1284-1292. doi:10.1097/01.MLR.0000093487.7 8664.3C

30. Staples LG, Dear BF, Gandy M, et al. Psychometric properties and clinical utility of brief measures of depression, anxiety, and general distress: the PHQ-2, GAD-2, and K-6. Gen Hosp Psychiatry. 2019;56 (November2018):13-18. doi:10.1016/j.genhosppsych.2018.11.003

31. Bennett IM, Coco A, Coyne JC, et al. Efficiency of a two-item pre-screen to reduce the burden of depression screening in pregnancy and postpartum: an IMPLICIT network study. J Am Board Fam Med. 2008;21(4):317-325. doi:10.3122/jabfm.2008.04.080048

32. First MB, Williams JBW, Karg RS, Spitzer RL. Structured Clinical Interview for DSM-5 ${ }^{\circledR}$ Disorders-Clinician Version (SCID-5-CV). Washington, DC: American Psychiatric Association. 2016.

33. Tingley D, Yamamoto T, Hirose K, Keele L, Imai K. Mediation: r Package for Causal Mediation Analysis. J Stat Softw. 2014;59 (5):1-38. doi:10.18637/jss.v059.i05

34. R Development Core Team. R: A Language and Environment for Statistical Computing. R Foundation for Statistical Computing; 2021.

35. Verreault N, Da Costa D, Marchand A, Ireland K, Dritsa M, Khalifé S. Rates and risk factors associated with depressive symptoms during pregnancy and with postpartum onset. J Psychosom Obstet Gynaecol. 2014;35(3):84-91. doi:10.3109/0167482X.20 14.947953

36. Liu X, Chen M, Wang Y, et al. Prenatal anxiety and obstetric decisions among pregnant women in Wuhan and Chongqing during the COVID-19 outbreak: a cross-sectional study. BJOG an Int J Obstet Gynaecol. 2020;127(10):1229-1240. doi:10.1111/14710528.16381

37. Salehi L, Rahimzadeh M, Molaei E, Zaheri H, Esmaelzadeh-Saeieh $\mathrm{S}$. The relationship among fear and anxiety of COVID-19, pregnancy experience, and mental health disorder in pregnant women: a structural equation model. Brain Behav. 2020;10(11):1-8. doi:10.1002/brb3.1835

38. Preis H, Mahaffey B, Heiselman C, Lobel M. Pandemic-Related Pregnancy Stress and Anxiety among Women Pregnant During the Coronavirus Disease 2019 Pandemic. American Journal of Obstetrics \& Gynecology MFM. 2020;2(3):100155. doi:10.1016/j.ajogmf.20 20.100155

39. Mazza C, Ricci E, Biondi S, et al. A nationwide survey of psychological distress among italian people during the covid-19 pandemic: immediate psychological responses and associated factors. Int J Environ Res Public Health. 2020;17(9):1-14. doi:10.3390/ ijerph17093165

40. Fan S, Guan J, Cao L, et al. Psychological effects caused by COVID-19 pandemic on pregnant women: a systematic review with meta-analysis. Asian J Psychiatry J. 2021;56. 
41. López-Morales H, Del Valle MV, Canet-Juric L, et al. Mental health of pregnant women during the COVID-19 pandemic: a longitudinal study. Psychiatry Res. 2021;295:295. doi:10.1016/j.psychres.20 20.113567

42. Gelaye B, Rondon MB, Araya R, Williams MA. Epidemiology of maternal depression, risk factors, and child outcomes in low-income and middle-income countries. The Lancet Psychiatry. 2016;3 (10):973-982. doi:10.1016/S2215-0366(16)30284-X

43. Lancaster CA, Gold KJ, Flynn HA, Yoo H, Marcus SM, Davis MM. Risk factors for depressive symptoms during pregnancy: a systematic review. Am J Obstet Gynaecol. 2010;202(1):5-14. doi:10.1016/j. ajog.2009.09.007.Risk

44. Brooks SK, Webster RK, Smith LE, et al. The psychological impact of quarantine and how to reduce it: rapid review of the evidence. Lancet. 2020;395(10227):912-920. doi:10.1016/S0140-6736(20) 30460-8

45. Mihashi M, Otsubo Y, Yinjuan X, Nagatomi K, Hoshiko M, Ishitake T. Predictive factors of psychological disorder development during recovery following SARS outbreak. Heal Psychol. 2009;28 (1):91-100. doi:10.1037/a0013674

46. Hawryluck L, Gold WL, Robinson S, Pogorski S, Galea S, Styra R. Psychological Effects of Quarantine for SARS. Emerg Infect Dis. 2004;10(7):1206-1212. doi:10.3201/eid1007.030703

47. Beutel ME, Klein EM, Brähler E, et al. Loneliness in the general population: prevalence, determinants and relations to mental health. BMC Psychiatry. 2017;17(1):1-7. doi:10.1186/s12888-017-1262-x

48. Ravaldi C, Ricca V, Wilson A, Homer C, Vannacci A. Previous psychopathology predicted severe COVID-19 concern, anxiety, and PTSD symptoms in pregnant women during "lockdown" in Italy. Arch Womens Ment Health. 2020;3. doi:10.1007/s00737-020-01086-0

49. Biaggi A, Conroy S, Pawlby S, Pariante CM. Identifying the women at risk of antenatal anxiety and depression: a systematic review. J Affect Disord. 2016;191:62-77. doi:10.1016/j.jad.2015.11.014

50. Davenport MH, Meyer S, Meah VL, Strynadka MC, Khurana R. Moms Are Not OK: COVID-19 and Maternal Mental Health. Front Glob Women's Heal. 2020;1(June):1-6. doi:10.3389/ fgwh.2020.00001

51. Zhang Y, Ma ZF. Psychological responses and lifestyle changes among pregnant women with respect to the early stages of COVID-19 pandemic. Int J Soc Psychiatry. 2020. doi:10.1177/ 0020764020952116

52. Wastnedge EA, Reynolds RM, van Boeckel SR, et al. Pregnancy and COVID-19. Physiol Rev. 2020;2507(1):1-9.

53. Lebel C, MacKinnon A, Bagshawe M, Tomfohr-Madsen L, Giesbrecht G. Elevated depression and anxiety symptoms among pregnant individuals during the COVID-19 pandemic. $J$ Affect Disord. 2020;277:5-13. doi:10.1016/j.jad.2020.07.126
54. Farewell CV, Jewell J, Walls J, Leiferman JA, Mixed-Methods Pilot A. Study of Perinatal Risk and Resilience During COVID-19. J Prim Care Community Heal. 2020;11. doi:10.1177/ 2150132720944074

55. Cotman CW, Berchtold NC. Exercise: a behavioral intervention to enhance brain health and plasticity. Trends Neurosci. 2002;25 (6):295-301. doi:10.1016/S0166-2236(02)02143-4

56. Wegner M, Helmich I, Machado S, Nardi AE, Arias-Carrión O, Budde H. Effects of Exercise on Anxiety and Depression Disorders: review of Meta-Analyses and Neurobiological Mechanisms. CNS Neurol Disord Targets. 2014;13:1002-1014. doi:10.2174/ 1871527313666140612102841

57. Lee CH, Huang N, Chang HJ, Hsu YJ, Wang MC, Chou YJ. The immediate effects of the severe acute respiratory syndrome (SARS) epidemic on childbirth in Taiwan. BMC Public Health. 2005;5:1-7. doi:10.1186/1471-2458-5-30

58. Ng J, Sham A, Tang PL, Fung S. SARS: pregnant women's fears and perceptions. Br J Midwifery. 2004;12(11):698-702. doi:10.12968/ bjom.2004.12.11.16710

59. Houston DM. Vulnerability to depressive mood reactions: retesting the hopelessness model of depression. Br J Soc Psychol. 1995;34 (3):293-302. doi:10.1111/j.2044-8309.1995.tb01065.x

60. Glover V. Maternal depression, anxiety and stress during pregnancy and child outcome; What needs to be done. Best Pract Res Clin Obstet Gynaecol. 2014;28(1):25-35. doi:10.1016/j.bpobgyn.20 13.08.017

61. Kinsella MT, Monk C. Impact of maternal stress, depression and anxiety on fetal neurobehavioral development. Clin Obstet Gynaecol. 2009;52(3):425-440. doi:10.1097/GRF.0b013e318 $1 \mathrm{~b} 52 \mathrm{df} 1$

62. Adamson B, Letourneau N, Lebel C. Prenatal maternal anxiety and children's brain structure and function: a systematic review of neuroimaging studies. J Affect Disord. 2018;241:117-126. doi:10.1016/j. jad.2018.08.029

63. House SJ, Tripathi SP, Knight BT, Morris N, Newport DJ, Stowe ZN. Obsessive-compulsive disorder in pregnancy and the postpartum period: course of illness and obstetrical outcome. Arch Womens Ment Health. 2015;19(1):3-10. doi:10.1007/s00737-015-0542-z

64. Coussons-Read ME. Effects of prenatal stress on pregnancy and human development: mechanisms and pathways. Obstet Med. 2013;6(2):52-57. doi:10.1177/1753495X12473751

65. Abdoli A, Falahi S, Kenarkoohi A, Shams M, Mir H, Jahromi MAM. The COVID-19 pandemic, psychological stress during pregnancy, and risk of neurodevelopmental disorders in offspring: a neglected consequence. J Psychosom Obstet Gynaecol. 2020;41(3):247-248. doi:10.1080/0167482X.2020.1761321

\section{Publish your work in this journal}

The International Journal of Women's Health is an international, peerreviewed open-access journal publishing original research, reports, editorials, reviews and commentaries on all aspects of women's healthcare including gynecology, obstetrics, and breast cancer. The manuscript management system is completely online and includes a very quick and fair peer-review system, which is all easy to use. Visit http://www.dovepress.com/testimonials.php to read real quotes from published authors. 